\title{
Stability of spheres under volume-preserving mean curvature flow
}

\author{
D. Antonopoulou, G. Karali, and I.M. Sigal \\ Communicated by Mikhail Vishik, received September 14, 2010.
}

\begin{abstract}
We give a new, elementary proof of the theorem, due to J. Escher and G. Simonett, that for the initial conditions close to Eucleadian spheres the solutions of the volume-preserving mean curvature flow converge to Eucleadian spheres (which, in general, differ from the initial spheres). Our result is in the metric given by Sobolev norms. While the proof by J. Escher and G. Simonett uses extensively rather involved results from the infinite-dimensional invariant manifold theory and quasilinear parabolic differential equations, our main point is to use an orthogonal decomposition of the solutions near the manifold of Euclidean spheres and differential inequalities for the Lyapunov functionals. Apart from local well-posedness, which is proven along standard lines, our proof is completely self-contained.
\end{abstract}

\section{Contents}

1. Introduction 328

Acknowledgement 329

2. Volume preserving flow for graphs 329

3. Differential equation for $\varrho \quad 329$

4. Linearized map 331

5. Orthogonal decomposition of solutions 332

6. Reparametrization of solutions 333

7. Lvapunov functional 334

8. Proof of Theorem 2 335

Appendix A. Appendix A: Proof of Lemma 14

Appendix B. Appendix B: Proof of Lemma 5

Appendix C. Appendix C: Expression for $\mathcal{N}(\xi) \quad 339$

Appendix D. Appendix D: Proof of 36 341

References 344

2000 Mathematics Subject Classification. Primary 35; Secondary 37.

Key words and phrases. Eucleadian sphere, volume-preserving mean curvature flow, Lyapunov functional. 


\section{Introduction}

In this paper we study the long time behavior of volume preserving mean curvature flow (VPMCF). This flow is a natural modification of the mean curvature flow (MCF) such that the volume enclosed by the evolving surface is preserved. Besides of an interest on its own, such a flow appears in material sciences as an interface dynamics in the case of the mass conservation (see e.g. $\mathbf{1 7}, \mathbf{5}, \mathbf{7}]$ and was used recently in Differential Geometry and General Relativity (13, 11). Given an initial simple, closed hypersurface $S_{0}$ in $\mathbb{R}^{n+1}$ the latter flow determines a family $\left\{S_{t} ; t \geq 0\right\}$ of smooth closed hypersurfaces in $\mathbb{R}^{n+1}$ satisfying the following evolution equation:

$$
V=h-H,
$$

where $V=V(t)$ denotes the normal velocity of $S_{t}$ at time $t$ and $H=H(t)$ stands for the mean curvature of $S_{t}$. Finally, $h=h(t)$ is the average of the mean curvature on $S_{t}$, i.e.,

$$
h:=\frac{\int_{S_{t}} H d \sigma}{\int_{S_{t}} d \sigma}, t \geq 0 .
$$

If $x=\sigma(u, t)$ is a parametrization of $S_{t}$ (or an immersion), then $V=\partial_{t} x \cdot \nu$, where $\nu$ is the unit normal vector field on $S_{t}$.

Like the MCF, the VPMCF shrinks the area of the surfaces, is invariant under rigid motions (translations and rotations) and appropriate scaling, but, unlike the MCF, the VPMCF has stationary solutions - Euclidean spheres (for closed surfaces) and cylinders for surfaces with flat boundaries.

The global well-posedness of VPMCF for smooth and uniformly convex initial conditions and for Hölder continuous initial conditions close to spheres was proven in [12] and [9], respectively. Results of this paper imply the global well-posedness for Sobolev initial conditions close to spheres.

G. Huisken ([12]), in the general case, and M. Gage (10]), for curves, proved that the solution to (11) exists globally and converges exponentially fast to a sphere, provided that the initial surface $S_{0}$ is uniformly convex and smooth. Moreover, it is shown in [12, 10 that $S_{t}$ stays uniformly convex for all $t \geq 0$. Athanassenas [3, 4 ] has shown neckpinching of certain class of rotationally symmetric surfaces under the volume preserving modification of the mean curvature flow. See also N. Alikakos and A. Freire [1. Later J. Escher and G. Simonett ([9]) proved, by means of a center manifold analysis, the asymptotic stability of spheres under Hölder norm (see also [15]).

In this paper we give a new, elementary proof of this theorem, in the metric given by Sobolev norms. (Though the proof in $\mathbf{9}$ is short and elegant it uses extensively rather involved results from the infinite-dimensional invariant manifold theory and quasilinear parabolic differential equations). Our main point is to use an orthogonal decomposition of the solutions near the manifold of Euclidean spheres and differential inequalities for the Lyapunov functionals. Apart from local wellposedness, which is proven along standard lines, see 12], our proof is completely self-contained. We believe our techniques can be extended to other flows, such as anisotropic volume preserving mean curvature flow ([2]), Mullins-Sekerka and Hele-Shaw models in the theory of phase transitions (see e.g. [8]). 
Let $\Gamma$ be the $n$-dimensional unit sphere in $\mathbb{R}^{n+1}$, centered at the origin, and let $H^{k}$ be the Sobolev space over $\Gamma$. Our main result is as follows.

Theorem 1. For initial conditions in $H^{k}$, for some $k>n / 2+1$, and close to Euclidean spheres, solutions of (10) exist globally and, as $t \rightarrow \infty$, converge exponentially in $H^{k}, k>n / 2+1$ to Euclidian spheres.

More precise formulation of Theorem 10 will be given in Section 2 Note that the initial conditions here do not have to be convex (the principal curvatures could be of either sign and arbitrary large in absolute value) and that the theorem implies that the VPMCF has no stationary solutions close to Euclidean spheres.

In Section 2 we give a precise formulation of Theorem 1 in terms of graphs over spheres. In Section 3 we find the equation for the graph function equivalent to (11). The proof of Theorem 1 is given in Sections 48 with some technical computations carried out in Appendices AD The latter appendices were worked out jointly with Wenbin Kong and are used also in [14.

\section{Acknowledgement}

The last author is grateful to Stephen Gustafson and Wenbin Kong for stimulating discussions. Research of G. Karali was supported by a Marie Curie International Reintegration Grant within the 7th European Community Framework Programme under the grant MIRG-CT-2007-200526, research of I.M. Sigal was supported by NSERC under Grant N7901.

\section{Volume preserving flow for graphs}

Let $\Gamma$ be the $n$-dimensional unit sphere in $\mathbb{R}^{n+1}$, centered at the origin, and let a hypersurface $S$ be a graph (in normal direction) over $\Gamma$, i.e. there exists a function $\rho: \Gamma \rightarrow \mathbb{R}$ such that

$$
\theta_{\rho}: \omega \mapsto \rho(\omega) \omega
$$

is a diffeomorphism from $\Gamma$ to $S$. We write $S=\operatorname{graph}_{\Gamma} \rho$.

Let $S_{R, z}$ denote the sphere of radius $R$, centered at $z \in \mathbb{R}^{n+1}, \alpha=(R, z)$ and $\rho_{\alpha}$ be the map from $\Gamma$ to $\mathbb{R}$ such that $\theta_{\rho_{\alpha}}(\omega)=\rho_{\alpha}(\omega) \omega$ is a diffeomorphism from $\Gamma$ to $S_{\alpha}$. Let $\alpha^{\prime}=(1,0)$, then $\rho_{\alpha^{\prime}} \equiv 1$. We give a more precise version to Theorem 1

THeorem 2. If the initial datum $S_{0}$ of equation (11) satisfies $S_{0}=\operatorname{graph}_{\Gamma} \rho_{0}$, with $\rho_{0} \in H^{k}(\Gamma)$ and $\left\|\rho_{0}-1\right\|_{H^{k}} \ll 1$ for some $k>\frac{n}{2}+1$, then (1) has a unique global solution, $S_{t}$, which is a graph over $\Gamma$ of $\rho(t) \in H^{k}(\Gamma)$ and which satisfies

$$
\left\|\rho(t)-\rho_{\alpha(t)}\right\|_{H^{k}} \lesssim e^{-\frac{\theta}{2} t}
$$

for some $\alpha(t)$ such that $\alpha(t) \rightarrow \alpha_{\infty}$ for some $\alpha_{\infty}$. Here $\theta>0$ is the 3rd smallest eigenvalue of the negative Laplace-Beltrami operator $-\Delta$ on $L^{2}(\Gamma)$.

\section{Differential equation for $\rho$}

In what follows $g_{i j}$ is the metric induced on $\Gamma$ by the inner product in $\mathbb{R}^{n+1}$ and $\Delta$ is the Laplace-Beltrami operator in this metric. In the local coordinates $u$ (with a local parametrization $x=x(u)$ ) we have $g_{i j}:=\frac{\partial x^{k}}{\partial u^{i}} \frac{\partial x^{k}}{\partial u^{j}}$. Let $\nabla$ and Hess $=\nabla^{2}$ be the standard connection on $\Gamma$ and the Hessian on $\Gamma$, respectively (see e.g. 16]). In components, $\operatorname{Hess\rho }(V, W)=V^{i} H e s s_{i j} \rho W^{j}$, where $H e s s_{i j}=\nabla_{i} \nabla_{j}$ and where the summation over the repeated indices is assumed. In local coordinates on $\Gamma$, 
$(H e s s \rho)_{i j}=\frac{\partial^{2} \rho}{\partial u^{i} \partial u^{j}}-\Gamma_{i j}^{k} \frac{\partial \rho}{\partial u^{k}}$, where $\Gamma_{i j}^{k}:=\frac{1}{2} g^{k n}\left(\frac{\partial g_{j n}}{\partial u^{i}}+\frac{\partial g_{i n}}{\partial u^{j}}-\frac{\partial g_{i j}}{\partial u^{n}}\right)$. We also identify $\nabla \rho$ with the gradient of $\rho$, with the components $\nabla^{k} \rho=g^{k m} \frac{\partial \rho}{\partial u^{m}}$.

Proposition 3. Let $S_{t}$ be a graph in normal direction over $\Gamma$, determined by the function $\rho(\cdot, t): \Gamma \rightarrow \mathbb{R}$. Then $S_{t}$ satisfies the (1) if and only if $\rho$ satisfies the equation

$$
\partial_{t} \rho=G(\rho)+g(\rho)
$$

with

$$
G(\rho)=\frac{1}{\rho^{2}} \Delta \rho-\frac{n}{\rho}-\frac{1}{\rho^{2} \mu^{2}(\rho)}\left(H e s s \rho(\nabla \rho, \nabla \rho)-\rho|\nabla \rho|^{2}\right),
$$

and

$$
g(\rho):=-\frac{\mu(\rho)}{\rho} \int_{\Gamma} G(\rho) \rho^{n} / \int_{\Gamma} \mu(\rho) \rho^{n-1},
$$

where

$$
\mu(\rho)=\sqrt{\rho^{2}+|\nabla \rho|^{2}} .
$$

Proof. Assume $S_{t}$ is a normal graph over $\Gamma$ determined by the function $\rho(\cdot, t)$ : $\Gamma \rightarrow \mathbb{R}$. We extend $\rho$ to $\mathbb{R}^{n+1} \backslash\{0\}$ by $\tilde{\rho}(x, t)=\rho(\hat{x}, t)=\rho(\alpha(x), t)$, where $\hat{x}=\frac{x}{|x|}$ and $\alpha: \mathbb{R}^{n+1} \rightarrow \Gamma, \alpha(x):=\hat{x}$. Then we can write $S_{t}=\left\{x \in \mathbb{R}^{n+1}: \varphi(x, t)=0\right\}$, where $\varphi(x, t)=|x|-\tilde{\rho}(x, t)$. (11) is equivalent to

$$
\partial_{t} \varphi=(\tilde{H}-h)\left|\nabla_{x} \varphi\right| \text { on } S_{t},
$$

where $\nabla_{x}$ is the usual gradient in $x$ and $\tilde{H}:=\operatorname{div}\left(\frac{\nabla_{x} \varphi}{\mid \nabla_{x} \varphi}\right)$ and his given in (2). We compute that $\partial_{t} \varphi=-\partial_{t} \tilde{\rho}, \nabla_{x} \varphi=\frac{x}{|x|}-\nabla_{x} \tilde{\rho}$ and

$$
\left|\nabla_{x} \varphi\right|=\sqrt{1+\left|\nabla_{x} \tilde{\rho}\right|^{2}}
$$

and therefore

$$
\partial_{t} \tilde{\rho}=\tilde{J}(\tilde{\rho}) \text { on } S_{t},
$$

where $\tilde{J}(\tilde{\rho})=\sqrt{1+\left|\nabla_{x} \tilde{\rho}\right|^{2}}(h-\tilde{H})$ with $\tilde{H}=\operatorname{div}\left(\frac{\frac{x}{|x|}-\nabla_{x} \tilde{\rho}}{\sqrt{1+\left|\nabla_{x} \tilde{\rho}\right|^{2}}}\right)$. Since $\tilde{\rho}(\lambda x)=\tilde{\rho}(x)$, we have that $x \cdot \nabla_{x} \tilde{\rho}=0$. Differentiating this equation with respect to $x_{i}$ we find that $x \cdot \nabla_{x} \partial_{x_{i}} \tilde{\rho}=-\partial_{x_{i}} \tilde{\rho}$, and therefore $x \cdot \nabla_{x}\left|\nabla_{x} \tilde{\rho}\right|^{2}=2\left|\nabla_{x} \tilde{\rho}\right|^{2}$. Using this relation, we compute

$$
\tilde{H}=\frac{\frac{n}{|x|}-\Delta_{x} \tilde{\rho}}{\sqrt{1+\left|\nabla_{x} \tilde{\rho}\right|^{2}}}+\frac{-\frac{1}{|x|}\left|\nabla_{x} \tilde{\rho}\right|^{2}+\nabla_{x} \tilde{\rho} \cdot \operatorname{Hess}_{x}(\tilde{\rho}) \nabla_{x} \tilde{\rho}}{\left(1+\left|\nabla_{x} \tilde{\rho}\right|^{2}\right)^{3 / 2}}
$$

where Hess $x_{x}:=\left(\partial_{x_{i}} \partial_{x_{j}}\right)$, the standard Hessian in $x$. Let $r=|x|$. We note first that due to the well-known representation (see [6])

$$
\Delta_{x}=r^{-n} \partial_{r} r^{n} \partial_{r}+\frac{1}{r^{2}} \Delta \text { on } \mathbb{R}^{n+1},
$$

we have that $\left.\Delta_{x} \tilde{\rho}\right|_{S_{t}}=\frac{1}{\tilde{\rho}^{2}} \Delta \rho$. Next, we need the following lemmas which is proved in Appendices $\mathrm{A}$ and $\mathrm{B}$ respectively: 
LEMMA 4.

$$
\begin{gathered}
\left|\nabla_{x} \tilde{\rho}\right|^{2}=\frac{1}{|x|^{2}}|\nabla \rho|^{2}, \\
\nabla_{x} \tilde{\rho} \cdot H e s s_{x} \tilde{\rho} \nabla_{x} \tilde{\rho}=\frac{1}{|x|^{4}} \operatorname{Hess} \rho(\nabla \rho, \nabla \rho) .
\end{gathered}
$$

Lemma 5. Write $s \in S$ as $s=\rho(\gamma) \gamma, \gamma \in \Gamma$. Then

$$
\int_{S_{t}} f=\int_{\Gamma} f(\rho(\cdot) \cdot) \mu(\rho) \rho^{n-1} .
$$

The first lemma and the equations $H=\left.\tilde{H}\right|_{S_{t}}$ and (11) give

$$
H(\rho):=\frac{-\rho}{\sqrt{\rho^{2}+|\nabla \rho|^{2}}} G(\rho) .
$$

and therefore $\left.\tilde{J}(\tilde{\rho})\right|_{S_{t}}=\frac{\mu(\rho)}{\rho} h+G(\rho)$. To compute $h$ we use in addition, the second lemma to obtain (6). This, together with (10), gives (44) - (6). Hence if $S_{t}=\operatorname{graph}_{\Gamma} \rho$ satisfies (11), then $\rho=\rho(t)$ satisfies (4) - (6). Reversing the steps we see that if $\rho$ satisfies (4) - (6), then $S_{t}=\operatorname{graph}_{\Gamma} \rho_{t}$ satisfies (11).

In the following we will consider equation (4) instead of equation (1).

\section{Linearized map}

In this section we study, on $L^{2}(\Gamma)$, the linear operator $L_{\alpha}=-\partial J\left(\rho_{\alpha}\right)$, which is the Gâteaux derivative of the map

$$
J(\rho):=G(\rho)+g(\rho),
$$

see (41), at the sphere $\rho_{\alpha}$. We begin with the easiest case of the linearization on a sphere of radius $R$ centered at the origin: $L_{R 0}:=-\partial J(R)\left(\rho_{R 0}=R\right)$. Using the definition (7) and the elementary relations $\left.\mu\right|_{\rho=R}=R,\left.\partial_{\rho} \mu\right|_{\rho=R}=1,\left.G\right|_{\rho=R}=-\frac{n}{R}$ and $\left.\partial_{\rho} G\right|_{\rho=R}=\frac{n}{R^{2}}$, we obtain (see [8])

$$
L_{R 0} \xi=-\frac{1}{R^{2}}(\Delta+n) \xi+\frac{n}{|\Gamma| R^{2}} \int_{\Gamma} \xi
$$

Proposition 6. $L_{R 0}$ is self-adjoint on $L^{2}(\Gamma)$ with discrete spectrum, $\sigma\left(L_{R 0}\right)$, accumulating at $+\infty$. Moreover, $\sigma\left(L_{R 0}\right) \subset[0,+\infty)$ and 0 is an eigenvalue of multiplicity $n+2$ with the eigenfunctions $\left\{1, \frac{x^{1}}{|x|}, \cdots, \frac{x^{n+1}}{|x|}\right\}$.

Proof. Since $\left\langle\eta, \int \xi\right\rangle=\int \bar{\eta} \int \xi=\int\left(\int \bar{\eta}\right) \xi=\left\langle\int \eta, \xi\right\rangle$ and since $\Delta$ is selfadjoint, we have that $L_{R 0}$ is self-adjoint as well. By the general fact that $\Gamma$ is compact, the spectrum of $L_{R 0}$ is discrete and accumulating at $+\infty$. Observe that $L_{R 0} \mathbf{1}=0$. Consider $L_{R 0}$ acting on $\mathbf{1}^{\perp}=\left\{\xi \in L^{2}(\Gamma): \int_{\Gamma} \xi=0\right\}$ and let $L_{R 0}^{\perp}=$ $\left.L_{R 0}\right|_{\left(\mathbf{1}^{\perp}\right)}$. Then $L_{R 0}^{\perp}=-\frac{1}{R^{2}}(\Delta+n)$. The spectrum of $-\Delta$ is well-known (see [18): $\{l(l+n-1), l=0,1, \cdots\}$, with the corresponding eigenspaces $H_{l}$ of the dimension $\operatorname{dim} H_{l}=\left(\begin{array}{c}n+l \\ n\end{array}\right)-\left(\begin{array}{c}n+l-2 \\ n\end{array}\right)$. Moreover, $H_{0}=\operatorname{span}\{1\}$ and $H_{1}=\operatorname{span}\left\{\frac{x^{1}}{|x|}, \cdots, \frac{x^{n+1}}{|x|}\right\}$. Hence the spectrum of $L_{R 0}$ is $\left\{0, \frac{1}{R^{2}}(l(l+n-1)-n)\right.$ : $l=2,3, \cdots\}$, with the zero eigenvalue of multiplicity $n+2$ having the eigenfunctions $\left\{1, \frac{x^{1}}{|x|}, \cdots, \frac{x^{n+1}}{|x|}\right\}$. Hence the proposition follows. 
This proposition implies the estimate

$$
\left\langle\xi, L_{R 0} \xi\right\rangle \geq \frac{n+2}{R^{2}}\|\xi\|^{2} \text { if } \xi \perp 1, \quad \frac{x^{1}}{|x|}, \cdots, \frac{x^{n+1}}{|x|} .
$$

A key fact in understanding the spectrum of the operator $L_{\alpha}$ is that $\partial_{\alpha} \rho_{\alpha}$ are zero modes of this operator:

$$
L_{\alpha} \partial_{\alpha} \rho_{\alpha}=0
$$

Indeed, this equation is obtained by differentiating $J\left(\rho_{\alpha}\right)=0$ we find $\partial J\left(\rho_{\alpha}\right) \partial_{\alpha} \rho_{\alpha}=$ 0 . These zero modes are related to the zero modes of the operator $L_{R 0}$ described in Proposition 6. The fact that $S_{\alpha}=\operatorname{graph} \rho_{\alpha}$ can be written as $\left|\rho_{\alpha}(\hat{x}) \hat{x}-z\right|=R$ implies that $\rho_{\alpha}(\hat{x})^{2}+|z|^{2}-2 \rho_{\alpha}(\hat{x}) z \cdot \hat{x}=R^{2}$ and therefore

$$
\rho_{\alpha}(\hat{x})=z \cdot \hat{x}+\sqrt{R^{2}-\left(z \cdot \hat{x}^{\perp}\right)^{2}} \text {, where }\left(z \cdot \hat{x}^{\perp}\right)^{2}:=|z|^{2}-(z \cdot \hat{x})^{2}
$$

and, recall, $\hat{x}=\frac{x}{|x|}$. Differentiating the former relation with respect to $R$ and $z^{j}$, we obtain

$$
\partial_{R} \rho_{\alpha}(\hat{x})=\frac{R}{\rho_{\alpha}(\hat{x})-z \cdot \hat{x}} \text { and } \partial_{z^{j}} \rho_{\alpha}(\hat{x})=\frac{\rho_{\alpha}(\hat{x}) \hat{x^{j}}-z^{j}}{\rho_{\alpha}(\hat{x})-z \cdot \hat{x}} .
$$

Hence we have that

$$
\partial_{R} \rho_{\alpha}(\hat{x})=1+O(|z|), \partial_{z^{j}} \rho_{\alpha}(\hat{x})=\hat{x}^{j}+O(|z|),
$$

which relates (20) to Proposition 6.

(20) shows that $\operatorname{Span}\left\{\partial_{\alpha} \rho_{\alpha}\right\} \subset$ Null $L_{\alpha}$. We conjecture that

$$
\operatorname{Span}\left\{\partial_{\alpha} \rho_{\alpha}\right\}=\operatorname{Null} L_{\alpha} .
$$

For $|z(\alpha)|$ sufficiently small, this conjecture follows from Proposition 6 by perturbation theory, using the following decomposition, which can be easily seen from the definition of $L_{\alpha}$,

$$
L_{\alpha}=L_{R 0}+M
$$

where $\alpha=(R, z)$ and where the 2 nd order operator $M$ satisfies the estimate $\|M \xi\| \leq$ $c|z|\left\|L_{R 0} \xi\right\|$.

\section{Orthogonal decomposition of solutions}

In what follows the inner product and orthogonality relation is understood in the sense of $L^{2}(\Gamma)$. We define the manifold of spheres as $\mathcal{M}=\left\{\rho_{\alpha}: \alpha \in \mathbb{R}^{+} \times \mathbb{R}^{n+1}\right\}$. We have $T_{\rho_{\alpha}} \mathcal{M}=\operatorname{span}\left\{\partial_{R} \rho_{\alpha}, \partial_{z^{j}} \rho_{\alpha}\right\}$. Thus $\hat{x}^{j}, j=0, \ldots, n+1$, where we denoted $\hat{x}^{0} \equiv 1$, span an approximate tangent space $T_{\rho_{\alpha}} \mathcal{M}$. Recall that $\alpha^{\prime}=(1,0)$ and $\rho_{\alpha^{\prime}} \equiv 1$.

Proposition 7. There is $\delta>0$, s.t. if $\left\|\rho-\rho_{\alpha^{\prime}}\right\| \leq \varepsilon$, then there exists $\alpha=\alpha(\rho)$ so that

$$
\rho-\rho_{\alpha} \perp \hat{x}^{j}, j=0, \ldots, n+1, \text { in } L^{2}(\Gamma, d \gamma) .
$$

Moreover, $\left|\alpha(\rho)-\alpha^{\prime}\right| \lesssim\left\|\rho-\rho_{\alpha^{\prime}}\right\|$ and $\left\|\rho-\rho_{\alpha(\rho)}\right\|_{H^{k}} \lesssim\left\|\rho-\rho_{\alpha^{\prime}}\right\|_{H^{k}} \forall k$. 
Proof. The orthogonality conditions on the fluctuation can be written as $F(\rho, \alpha)=0$, where $F: L^{2}(\Gamma) \times \mathbb{R}^{+} \times \mathbb{R}^{n+1} \rightarrow \mathbb{R}^{n+1}$ is defined as $F(\rho, \alpha)=$ $\left\langle\rho-\rho_{\alpha}, \hat{x}^{j}\right\rangle$. Here and in what follows, all inner products are the $L^{2}$ inner products.

Note first that the mapping $F$ is $C^{\infty}$ and $F\left(\rho_{\alpha}, \alpha\right)=0, \forall \alpha$. We claim that the linear map $\left.\partial_{\alpha} F(\rho, \alpha)\right|_{\rho=\rho_{\alpha}}$ is invertible, provided $|z|$, where $(R, z)=\alpha$, is sufficiently small. Indeed, let $\alpha^{0}=R, \alpha^{j}=z^{j}, j=1, \ldots, n+1$. We compute using (22) that

$$
\left.\partial_{\alpha^{i}} F(\rho, \alpha)\right|_{\rho=\rho_{\alpha}}=-\left\langle\partial_{\alpha^{i}} \rho_{\alpha}, \hat{x}^{j}\right\rangle=-\left\langle\hat{x}^{i}, \hat{x}^{j}\right\rangle+O(|z|) .
$$

Since $\left\langle\hat{x}^{i}, \hat{x}^{j}\right\rangle$ is a diagonal matrix $\operatorname{diag}\left(-\int_{\Gamma} 1,-\int_{\Gamma}\left(\hat{x}^{1}\right)^{2}, \cdots,-\int_{\Gamma}\left(\hat{x}^{n+1}\right)^{2}\right)$ we have that $\left.\partial_{\alpha} F(\rho, \alpha)\right|_{\rho=\rho_{\alpha}}$ is invertible, if $|z|$ is sufficiently small. Recall that $\alpha^{\prime}=(R, 0)$. Hence, $|z| \leq\left|\alpha-\alpha^{\prime}\right|$ and is small, if $\left|\alpha-\alpha^{\prime}\right|$ is small. Thus, the first part of the proposition follows by the implicit function theorem.

Next we expand the function $F(\rho, \alpha)$ in $\alpha$ around $\alpha^{\prime}$ :

$$
F(\rho, \alpha)=F\left(\rho, \alpha^{\prime}\right)+\partial_{\alpha} F\left(\rho, \alpha^{\prime}\right)\left(\alpha-\alpha^{\prime}\right)+R(\rho, \alpha),
$$

where $R(\rho, \alpha)=O\left(\left|\alpha-\alpha^{\prime}\right|^{2}\right)$ uniformly in $\alpha$ and $\rho$ for $\rho$ in a small neighbourhood of $\rho_{\alpha}$. Hence we have $\left|\alpha-\alpha^{\prime}\right| \lesssim F\left(\rho, \alpha^{\prime}\right)+\left|\alpha-\alpha^{\prime}\right|^{2}$. Since $\left|F\left(\rho, \alpha^{\prime}\right)\right| \lesssim\left\|\rho-\rho_{\alpha^{\prime}}\right\| \ll$ 1 , it follows that $\left|\alpha-\alpha^{\prime}\right| \lesssim\left|F\left(\rho, \alpha^{\prime}\right)\right| \lesssim\left\|\rho-\rho_{\alpha^{\prime}}\right\|$. The latter estimate, the triangle inequality, $\left\|\rho-\rho_{\alpha(\rho)}\right\|_{H^{k}} \lesssim\left\|\rho-\rho_{\alpha^{\prime}}\right\|_{H^{k}}+\left\|\rho_{\alpha^{\prime}}-\rho_{\alpha(\rho)}\right\|_{H^{k}}$ and the estimate $\left\|\rho_{\alpha^{\prime}}-\rho_{\alpha}\right\|_{H^{k}} \lesssim\left|\alpha^{\prime}-\alpha\right|$ imply the last inequality of the proposition.

\section{Reparametrization of solutions}

Applying Proposition 7 to the solution $\rho(t)$ we find $\alpha(t)$ s.t.

$$
\rho(\gamma, t)=\rho_{\alpha(t)}(\gamma)+\xi(\gamma, t),
$$

where $\xi \perp \hat{x^{j}}, j=0, \ldots, n+1$, as long as $\left\|\rho(t)-\rho_{\alpha^{\prime}}\right\| \leq \delta$. Plug (25) into (4) to obtain

$$
\partial_{t} \xi=-L_{\alpha} \xi+\mathcal{N}(\xi)+\mathcal{F}
$$

where

$$
\begin{aligned}
& L_{\alpha}=-\partial J\left(\rho_{\alpha}\right), \\
& \mathcal{N}(\xi)=J\left(\rho_{\alpha}+\xi\right)-J\left(\rho_{\alpha}\right)-\partial J\left(\rho_{\alpha}\right) \xi, \\
& \mathcal{F}=-\partial_{\alpha} \rho_{\alpha} \cdot \dot{\alpha} .
\end{aligned}
$$

Now, we project (26) onto $\operatorname{span}\left\{\hat{x}^{j}, j=0, \ldots, n+1\right\}$. By $\xi \perp \hat{x}^{j}$ and $L_{R 0} \partial_{\alpha^{j}} \hat{x}^{j}=$ $0, j=0, \ldots, n+1$, we have

$$
\begin{aligned}
& -\left\langle\partial_{t} \xi, \hat{x}^{j}\right\rangle=-\left\langle\xi, \partial_{t} \hat{x}^{j}\right\rangle=0, \\
& \text { - }\left\langle L_{\alpha} \xi, \hat{x}^{j}\right\rangle=\left\langle\xi, L_{\alpha} \hat{x}^{j}\right\rangle=\left\langle\xi, M \hat{x}^{j}\right\rangle, \\
& \text { - }\left\langle\mathcal{F}, \hat{x}^{j}\right\rangle=-\sum_{i} \dot{\alpha}^{i}\left\langle\partial_{\alpha^{i}} \rho_{\alpha}, \hat{x}^{j}\right\rangle .
\end{aligned}
$$

Then we obtain $\Omega \dot{\alpha}=\left\langle\mathcal{N}(\xi), \partial_{\alpha} \rho_{\alpha}\right\rangle-\left\langle\xi, M \hat{x^{j}}\right\rangle$, where $\Omega$ is the matrix with the entries $\Omega^{i j}=\left\langle\partial_{\alpha^{i}} \rho_{\alpha}, \hat{x}^{j}\right\rangle, i, j=0,1, \cdots, n+1$. By (22), we know that $\Omega^{i j}=$ $\left\langle\hat{x}^{i}, \hat{x}^{j}\right\rangle+O(|z|)$. Assume $|z| \ll 1$. Then by the proof of Proposition $\mathbf{7}$ we know that $\Omega$ is invertible. This gives us

$$
\dot{\alpha}=\Omega^{-1}\left(\left\langle\mathcal{N}(\xi), \partial_{\alpha} \rho_{\alpha}\right\rangle-\langle\xi, M \hat{x}\rangle\right),
$$

which implies

$$
|\dot{\alpha}| \lesssim\|\mathcal{N}(\xi)\|_{1}+|z|\|\xi\|_{1}
$$


Next, we estimate $\mathcal{N}(\xi)$, defined in (27), with (17), (15) and (6). An explicit expression for $\mathcal{N}(\xi)$ is rather long and is given in Appendix C Here we write out only the worst term:

$$
-\frac{\operatorname{Hess}(\xi)(\nabla \xi, \nabla \xi)}{\rho^{2}\left(\rho^{2}+|\nabla \xi|^{2}\right)}
$$

where, recall, $\rho=\rho_{\alpha}+\xi$. Hence, assuming that $|\xi| \leq \frac{1}{2} \rho_{\alpha}$, we have that

$$
\|\mathcal{N}(\xi)\|_{1} \lesssim\left(\|\nabla \xi\|_{L^{4}}^{2}+\|\xi\|_{L^{2}}\right)\|\xi\|_{H^{2}}+\|\xi\|_{H^{1}}^{2}
$$

This together with (28) gives

$$
|\dot{\alpha}| \lesssim\left(\|\nabla \xi\|_{L^{\infty}}^{2}+\|\xi\|_{H^{1}}\right)\|\xi\|_{H^{2}}+|z|\|\xi\|_{1} .
$$

\section{Lyapunov functional}

In this section we assume that Proposition 7 holds and therefore the solution $\rho$ can be written as $\rho=\rho_{\alpha}+\xi$, with $\xi \perp 1, \frac{x^{1}}{\mid x}, \cdots, \frac{x^{n+1}}{|x|}$ in $L^{2}(\Gamma)$. Let $\alpha=(R, z)$. For $k \geq 1$, we define the functional $\Lambda_{k}(\xi)=\frac{1}{2}\left\langle\xi, L_{R 0}^{k} \xi\right\rangle$, where $L_{R 0}$ is given by (18) (i.e. $\left.L_{R 0} \xi=-\frac{1}{R^{2}}(\Delta+n) \xi+\frac{n}{\Gamma \mid R^{2}} \int_{\Gamma} \xi\right)$ and, recall, the inner product is taken in $L^{2}(\Gamma)$. Since $\xi \perp 1, \frac{x^{1}}{|x|}, \cdots, \frac{x^{n+1}}{|x|}$, we have, by (19), that $\left\langle L_{R 0} \xi, \xi\right\rangle \geq \frac{n+2}{R^{2}}\|\xi\|^{2}$.

Proposition 8. There exist constants $c>0$ and $C>0$ such that

$$
c R^{-2 k}\|\xi\|_{H^{k}}^{2} \leq \Lambda_{k}(\xi) \leq C R^{-2 k}\|\xi\|_{H^{k}}^{2} .
$$

Proof. By a standard computation, we see that there exists a $C>0$ such that $\left\langle\xi, L_{R 0}^{k} \xi\right\rangle \leq C R^{-2 k}\|\xi\|_{H^{k}}^{2}$. We prove the lower bound below. Recall $\left\langle\xi, L_{R 0} \xi\right\rangle \geq$ $\frac{n+2}{R^{2}}\|\xi\|^{2}$. From the definition of $L_{R 0}$ we also have $\left\langle\xi, L_{R 0} \xi\right\rangle=C_{1} R^{-2}\|\nabla \xi\|^{2}-$ $C_{2} R^{-2}\|\xi\|^{2}$ for some $C_{1}>0$ and $C_{2}>0$. These two inequalities imply that

$$
\begin{aligned}
\left\langle\xi, L_{R 0} \xi\right\rangle & =\lambda\left\langle\xi, L_{R 0} \xi\right\rangle+(1-\lambda)\left\langle\xi, L_{R 0} \xi\right\rangle \\
& \geq \lambda C_{1} R^{-2}\|\nabla \xi\|^{2}-\lambda C_{2} R^{-2}\|\xi\|^{2}+(1-\lambda)(n+2) R^{-2}\|\xi\|^{2} \\
& =\lambda C_{1} R^{-2}\left(\|\nabla \xi\|^{2}+\|\xi\|^{2}\right),
\end{aligned}
$$

provided that $\lambda=\frac{C}{C+C_{1}+C_{2}}$, where $C=n+2$.

For the general case, observe that $L_{R 0}$ is a self-adjoint operator and $L_{R 0}^{k}$ has the same eigenfunctions as $L_{R 0}$ with eigenvalues $\left\{\frac{1}{R^{2 k}}(l(l+n-1)-n)^{k}: l=0,1, \cdots\right\}$. Hence, by (19), $\left\langle\xi, L_{R 0}^{k} \xi\right\rangle \geq\left(\frac{n+2}{R^{2}}\right)^{k}\|\xi\|^{2}$. On the other hand, we have as before $\left\langle\xi, L_{R 0}^{k} \xi\right\rangle \geq\left(\frac{n}{R^{2}}\right)^{k}\left[\|\xi\|_{H^{k}}^{2}-C\|\xi\|^{2}\right]$. Then proceeding as before we find $\left\langle\xi, L_{R 0}^{k} \xi\right\rangle \gtrsim$ $R^{-2 k}\|\xi\|_{H^{k}}^{2}$, which is the lower bound in the proposition.

Proposition 9. Let $k>\frac{n}{2}+1,|z| \ll 1$ and $|\xi| \leq \frac{1}{2} \rho_{\alpha}$. Then there exists a constant $C>0$ such that

$$
\partial_{t} \Lambda_{k}(\xi) \leq-\frac{n+2}{R^{2}} \Lambda_{k}(\xi)-\left[\frac{1}{3}-C\left(\Lambda_{k}(\xi)^{1 / 2}+\Lambda_{k}(\xi)^{k}\right)\right]\left\|L_{R 0}^{\frac{k+1}{2}} \xi\right\|^{2} .
$$

Proof. We have $\frac{1}{2} \partial_{t}\left\langle\xi, L_{R 0}^{k} \xi\right\rangle=\left\langle\partial_{t} \xi, L_{R 0}^{k} \xi\right\rangle+\frac{1}{2}\left\langle\xi,\left(\partial_{t} L_{R 0}^{k}\right) \xi\right\rangle$. Now, using (26), we obtain

(33) $\frac{1}{2} \partial_{t}\left\langle\xi, L_{R 0}^{k} \xi\right\rangle=-\left\langle L_{\alpha} \xi, L_{R 0}^{k} \xi\right\rangle+\left\langle\mathcal{N}(\xi), L_{R 0}^{k} \xi\right\rangle+\left\langle\mathcal{F}, L_{R 0}^{k} \xi\right\rangle+\frac{1}{2}\left\langle\xi,\left(\partial_{t} L_{R 0}^{k}\right) \xi\right\rangle$. 
We consider each term on the right hand side. First, we observe that one can show readily that the operator $M$ in the decomposition (24), $L_{\alpha}=L_{R 0}+M$, satisfies the estimate

$$
\left\|L_{R 0}^{\frac{k-1}{2}} M \xi\right\| \leq c|z|\left\|L_{R 0}^{\frac{k+1}{2}} \xi\right\| .
$$

Using this estimate and the lower bound (19), we obtain

$$
\begin{aligned}
& \left\langle L_{\alpha} \xi, L_{R 0}^{k} \xi\right\rangle \\
= & \frac{1}{2}\left\|L_{R 0}^{\frac{k+1}{2}} \xi\right\|^{2}+\frac{1}{2}\left\langle L_{R 0}^{\frac{k}{2}} \xi, L_{R 0} L_{R 0}^{\frac{k}{2}} \xi\right\rangle+\left\langle L_{R 0}^{\frac{k-1}{2}} M \xi, L_{R 0}^{\frac{k+1}{2}} \xi\right\rangle \\
\geq & \frac{1}{2}\left\|L_{R 0}^{\frac{k+1}{2}} \xi\right\|^{2}+\frac{n+2}{2 R^{2}}\left\langle L_{R 0}^{\frac{k}{2}} \xi, L_{R 0}^{\frac{k}{2}} \xi\right\rangle-c|z|\left\|L_{R 0}^{\frac{k+1}{2}} \xi\right\|^{2} \\
\geq & \frac{1}{3}\left\|L_{R 0}^{\frac{k+1}{2}} \xi\right\|^{2}+\frac{n+2}{R^{2}} \Lambda_{k}(\xi) .
\end{aligned}
$$

To estimate the next term we need the following inequality proven in Appendix B:

$$
\left\|L_{R 0}^{\frac{k-1}{2}} \mathcal{N}(\xi)\right\| \lesssim\left(\Lambda_{k}^{1 / 2}(\xi)+\Lambda_{k}^{k}(\xi)\right)\left\|L_{R 0}^{\frac{k+1}{2}} \xi\right\| .
$$

This estimate implies that

$$
\begin{aligned}
\left|\left\langle\mathcal{N}(\xi), L_{R 0}^{k} \xi\right\rangle\right| & =\left|\left\langle L_{R 0}^{\frac{k-1}{2}} \mathcal{N}(\xi), L_{R 0}^{\frac{k+1}{2}} \xi\right\rangle\right| \\
& \leq\left\|L_{R 0}^{\frac{k-1}{2}} \mathcal{N}(\xi)\right\|\left\|L_{R 0}^{\frac{k+1}{2}} \xi\right\| \\
& \leq C\left(\Lambda_{k}^{1 / 2}(\xi)+\Lambda_{k}^{k}(\xi)\right)\left\|L_{R 0}^{\frac{k+1}{2}} \xi\right\|^{2} .
\end{aligned}
$$

We have by (27),

$$
\left|\left\langle\mathcal{F}, L_{R 0}^{k} \xi\right\rangle\right|=|\dot{\alpha}|\left|\left\langle\partial_{\alpha} \rho_{\alpha}, L_{R 0}^{k} \xi\right\rangle\right|=|\dot{\alpha}|\left|\left\langle L_{R 0}^{k} \partial_{\alpha} \rho_{\alpha}, \xi\right\rangle\right| .
$$

Next, we use (31), the relation $L_{R 0} \partial_{\alpha} \rho_{\alpha}=M \partial_{\alpha} \rho_{\alpha}$ and (34) to obtain

$$
\left|\left\langle\mathcal{F}, L_{R 0}^{k} \xi\right\rangle\right| \leq C\left(\|\nabla \xi\|_{L^{\infty}}^{2}+\|\xi\|_{H^{1}}+|z|\right)\|\xi\|_{H^{2}}\|\xi\|_{1} .
$$

Finally, using (18) and (31), we obtain

$$
\left|\left\langle\xi,\left(\partial_{t} L_{R 0}^{k}\right) \xi\right\rangle\right|=\left|\frac{2 k \dot{R}}{R}\left\langle\xi, L_{R 0}^{k} \xi\right\rangle\right| \leq C\left(\|\nabla \xi\|_{L^{\infty}}^{2}+\|\xi\|_{H^{1}}+|z|\right)\|\xi\|_{H^{2}}\left\|L_{R 0}^{\frac{k}{2}} \xi\right\|^{2} .
$$

Now, by the condition $k>\frac{n}{2}+1$ and Proposition 8 we have that $\|\nabla \xi\|_{L^{\infty}}$, $\|\xi\|_{H^{2}},\|\xi\|_{H^{k}} \leq C \Lambda_{k}^{1 / 2}(\xi)$. This, together with (33), (35), (37), (38) and (39) and the condition $|z| \ll 1$, gives (32).

\section{Proof of Theorem 2}

First, we note that we can either assume that the initial conditions are smooth and use the proof of [12] of local well-posedness of (10) or we can adapt the latter proof to the Sobolev spaces used here.

We begin with an estimate of $|z|$ and $\left|\alpha-\alpha^{\prime}\right|$ (recall, that $\alpha=(R, z)$ and $\left.\alpha^{\prime}=(1,0)\right)$ in terms of the Lyapunov functionals $\Lambda_{k}(\xi)$. Using the estimates $\left|\partial_{t}\right| z|| \leq|\dot{z}| \leq|\dot{\alpha}|$ and $\|\nabla \xi\|_{L^{\infty}},\|\xi\|_{H^{2}},\|\xi\|_{H^{k}} \leq C_{1} \Lambda_{k}^{1 / 2}(\xi)$ and Eqn (31), we obtain

$$
\left|\partial_{t}\right| z|| \leq C_{2}\left[\Lambda_{k}(\xi)+\Lambda_{k}^{1 / 2}(\xi)+|z|\right] \Lambda_{k}^{1 / 2}(\xi)
$$

By Gronwall's inequality the equation (40) implies

$$
|z(t)| \leq C_{3} e^{C_{4} \int_{0}^{t} \Lambda_{k}^{1 / 2}(\xi(s)) d s}\left(\left|z_{0}\right|+\int_{0}^{t} \max \left(\Lambda_{k}^{3 / 2}(\xi(r)), \Lambda_{k}(\xi(r)) d r\right) .\right.
$$


Finally, by (31) we have that $|\dot{\alpha}| \leq C_{5}\left[\Lambda_{k}(\xi)+\Lambda_{k}^{1 / 2}(\xi)+|z|\right] \Lambda_{k}^{1 / 2}(\xi)$ and therefore

$$
\left|\alpha-\alpha^{\prime}\right| \leq\left|\alpha_{0}-\alpha^{\prime}\right|+C_{5}\left[\Lambda_{k}(\xi)+\Lambda_{k}^{1 / 2}(\xi)+|z|\right] \Lambda_{k}^{1 / 2}(\xi) .
$$

Let $\delta$ be the same as in Proposition 7 and let $\varepsilon$ be s.t. Proposition 9 holds for $|z| \leq \varepsilon$. The initial condition, $\xi_{0}$, for $\xi$ is given in Proposition 7 with $\rho=\rho_{0}$. By the latter proposition we can take initial condition $\rho_{0}$ such that $\Lambda_{k}\left(\xi_{0}\right)^{\frac{1}{2}}+\Lambda_{k}\left(\xi_{0}\right)^{k} \leq$ $\frac{1}{10 C}$, where the constant $C$ is the same as in Proposition $9 \Lambda_{k}\left(\xi_{0}\right)+\left|z_{0}\right| \leq \frac{1}{4 C^{\prime}} \varepsilon$, where $C^{\prime}=C_{3} e^{C_{4}}$, and $\left|\alpha_{0}-\alpha^{\prime}\right|+\left(1+C_{5} \frac{2 R^{2}}{n+2}\right) \Lambda_{k}^{1 / 2}\left(\xi_{0}\right) \leq \frac{1}{4} \delta$, with the constants $C_{3}, C_{4}$ and $C_{5}$, the same as in (41) and (42). Let

$T=\sup \left\{t>0: \Lambda_{k}(\xi(t))^{\frac{1}{2}}+\Lambda_{k}(\xi(t))^{k} \leq \frac{1}{5 C},|z(t)| \leq \frac{1}{2} \varepsilon, \Lambda_{k}(\xi(t))^{\frac{1}{2}}+\left|\alpha(t)-\alpha^{\prime}\right| \leq \frac{1}{2} \delta\right\}$.

Clearly, $T>0$ while we assume $T<\infty$. Then for any $t \leq T$ we get $\partial_{t} \Lambda_{k}(\xi) \leq$ $-\frac{n+2}{R^{2}} \Lambda_{k}(\xi)$. Integrate this:

$$
\Lambda_{k}(\xi) \leq \Lambda_{k}\left(\xi_{0}\right) e^{-\frac{n+2}{R^{2}} t}
$$

which implies

$$
\Lambda_{k}(\xi(T))^{\frac{1}{2}}+\Lambda_{k}(\xi(T))^{k} \leq \Lambda_{k}\left(\xi_{0}\right)^{\frac{1}{2}}+\Lambda_{k}\left(\xi_{0}\right)^{k} \leq \frac{1}{10 C} .
$$

and, together with 411,

$$
|z(t)| \leq C^{\prime}\left(\left|z_{0}\right|+\Lambda_{k}\left(\xi_{0}\right)\right) \leq \frac{1}{4} \varepsilon,
$$

uniformly in $t$. Finally, Eqns (42), (43) and (45) imply that

$$
\Lambda_{k}(\xi(t))^{\frac{1}{2}}+\left|\alpha-\alpha^{\prime}\right| \leq \frac{1}{4} \delta .
$$

This, together with (44) and (45), contradicts the assumption $T<\infty$, so $T=\infty$ and (44), (45) and (46) are valid for all $t<\infty$.

By (31) and (43) we have that $|\dot{\alpha}| \lesssim\left[\Lambda_{k}(\xi)+\Lambda_{k}^{1 / 2}(\xi)+|z|\right] \Lambda_{k}^{1 / 2}(\xi) \lesssim e^{-\frac{n+2}{2 R^{2}} t} \Lambda_{k}^{1 / 2}\left(\xi_{0}\right)$. Hence there exists $\alpha_{\infty}>0$ such that $\left|\alpha(t)-\alpha_{\infty}\right| \lesssim e^{-\frac{n+2}{2 R^{2}} t} \Lambda_{k}^{1 / 2}\left(\xi_{0}\right)$. To sum up we have $\rho(\gamma, t)=\rho_{\alpha(t)}(\gamma)+\xi(\gamma, t)$ and, by Proposition 8 and 433), $\|\xi(t)\|_{H^{k}} \lesssim$ $e^{-\frac{n+2}{2 R^{2}} t}\|\xi(0)\|_{H^{k}}$ and $\left|\alpha(t)-\alpha_{\infty}\right| \lesssim e^{-\frac{n+2}{2 R^{2}} t}\|\xi(0)\|_{H^{k}}$ for some $\alpha_{\infty}>0$. Due to the definition [25], this proves Theorem 2

\section{Appendix A. Appendix A: Proof of Lemma 4}

Let $\beta: U \rightarrow \mathbb{R}^{n+1}$ be a local parametrization of $\Gamma$, and we denote $\rho$ in the local coordinates, $\rho \circ \beta$, again as $\rho: U \rightarrow \mathbb{R}$. We write $\tilde{\rho}:=\rho \circ \alpha=\rho \circ \beta \circ \beta^{-1} \circ \alpha$, which we rewrite as $\tilde{\rho}=\rho \circ \sigma$, where $\sigma:=\beta^{-1} \circ \alpha: \mathbb{R}^{n+1} \rightarrow U$. Now, writing $u=u(x) \equiv \sigma(x)$, we define $\frac{\partial u^{k}}{\partial x^{2}} \frac{\partial u^{l}}{\partial x^{i}}=: \tilde{g}^{k l}$, where we use the convention of summing over repeated indices. We claim

$$
\tilde{g}_{i j}(x) g^{j k}(u)=\frac{1}{|x|^{2}} \delta_{i k} .
$$

Indeed, since $\beta(\sigma(x))=\alpha(x)$, we have

$$
\left(\frac{\partial x^{i}}{\partial u^{m}} \circ \sigma\right) \frac{\partial u^{m}}{\partial x^{j}}=\frac{\partial \alpha^{i}}{\partial x^{j}}=\frac{1}{|x|}\left(\delta_{i j}-\frac{x^{i} x^{j}}{|x|^{2}}\right) .
$$


Note that $\sigma$ is homogeneous of degree 0 , so $x \cdot \nabla_{x} \sigma=0$. This together with (48) implies that

$$
\begin{aligned}
& \tilde{g}^{i j}(x) g_{j k}(u)=\frac{\partial u^{i}}{\partial x^{m}} \frac{\partial u^{j}}{\partial x^{m}}\left(\frac{\partial x^{n}}{\partial u^{j}} \circ \sigma\right)\left(\frac{\partial x^{n}}{x^{k}} \circ \sigma\right) \\
& =\frac{1}{|x|} \frac{\partial u^{i}}{\partial x^{m}}\left(\delta_{m n}-\frac{x^{m} x^{n}}{|x|^{2}}\right) \frac{\partial x^{n}}{\partial u^{k}}=\frac{1}{|x|} \frac{\partial u^{i}}{\partial x^{m}} \frac{\partial x^{n}}{\partial u^{k}} .
\end{aligned}
$$

Since $|x| \frac{\partial u^{i}}{\partial x^{m}}$ is homogeneous of degree 0, we have that $|x| \frac{\partial u^{i}}{\partial x^{m}}=\left.\frac{\partial u^{i}}{\partial x^{m}}\right|_{\Gamma}$, and therefore $\frac{\partial u^{i}}{\partial x^{m}}=\frac{1}{|x|}\left(\left.\frac{\partial u^{i}}{\partial x^{m}}\right|_{\Gamma}\right)$. Using $\sigma \circ \beta=1_{U}$ we compute that $\left(\frac{\partial \sigma^{i}}{\partial x^{j}} \circ \beta\right) \frac{\partial \beta^{j}}{\partial u^{k}}=\delta_{i k}$, which is equivalent to $\left.\frac{\partial u^{i}}{\partial x^{j}}\right|_{\Gamma}\left(\frac{\partial x^{j}}{\partial u^{k}} \circ \sigma\right)=\delta_{i k}$. This gives us

$$
\frac{\partial u^{i}}{\partial x^{m}} \frac{\partial x^{m}}{\partial u^{k}}=\frac{1}{|x|}\left(\left.\frac{\partial u^{i}}{\partial x^{m}}\right|_{\Gamma}\right) \frac{\partial x^{m}}{\partial u^{k}}=\frac{1}{|x|} \delta_{i k} .
$$

From (49) and (50) we have the equation (47).

In what follows we use the relations $\frac{\partial \rho}{\partial u^{i}}=g_{i j} \nabla^{j} \rho$ (this follows from the definition of $\nabla \rho)$ and $\partial_{x^{i}} \tilde{\rho}=\frac{\partial u^{j}}{\partial x^{i}} \frac{\partial \rho}{\partial u^{j}}$. Using these relations and (47) we can compute

$$
\begin{aligned}
& \left|\nabla_{x} \tilde{\rho}\right|^{2}=\partial_{x^{i}} \tilde{\rho} \partial_{x^{i}} \tilde{\rho} \\
= & \frac{\partial u^{k}}{\partial x^{2}} \frac{\partial u^{l}}{\partial x^{i}} \partial_{u^{k}} \tilde{\rho} \partial_{u^{l}} \tilde{\rho}=\tilde{g}^{k l}(x) \partial_{u^{k}} \rho \partial_{u^{l}} \rho \\
= & \tilde{g}_{k l}(x) g_{k m}(u) \nabla^{m} \rho g_{l n}(u) \nabla^{n} \rho=\frac{1}{|x|^{2}} \nabla^{l} \rho g_{l n} \nabla^{n} \rho \\
= & \frac{1}{|x|^{2}}|\nabla \rho|^{2} .
\end{aligned}
$$

This gives (13).

Now we prove (14). We have

$$
\begin{aligned}
& \nabla_{x} \tilde{\rho} \cdot \operatorname{Hess}_{x}(\tilde{\rho}) \nabla_{x} \tilde{\rho}=\partial_{x^{i}} \tilde{\rho} \partial_{x^{i} x^{j}}^{2} \tilde{\rho} \partial_{x^{j}} \tilde{\rho} \\
= & \frac{\partial u^{m}}{\partial x^{i}} \frac{\partial \rho}{\partial u^{m}} \frac{\partial u^{l}}{\partial x^{i}} \frac{\partial}{\partial u^{l}}\left(\frac{\partial u^{k}}{\partial x^{j}} \frac{\partial \rho}{\partial u^{k}}\right) \frac{\partial u^{n}}{\partial x^{j}} \frac{\partial \rho}{\partial u^{n}} \\
= & \tilde{g}^{m l} \frac{\partial \rho}{\partial u^{m}} \frac{\partial}{\partial u^{l}}\left(\frac{\partial u^{k}}{\partial x^{j}} \frac{\partial \rho}{\partial u^{k}}\right) \frac{\partial u^{n}}{\partial x^{j}} \frac{\partial \rho}{\partial u^{n}} \\
= & \tilde{g}^{m l} \tilde{g}^{k n} \frac{\partial \rho}{\partial u^{m}} \frac{\partial^{\rho} \rho}{\partial u^{l} u^{k}} \frac{\partial \rho}{\partial u^{n}}+\tilde{g}^{m l} \frac{\partial \rho}{\partial u^{m}} \frac{\partial}{\partial u^{l}}\left(\frac{\partial u^{k}}{\partial x^{j}}\right) \frac{\partial \rho}{\partial u^{k}} \frac{\partial u^{n}}{\partial x^{j}} \frac{\partial \rho}{\partial u^{n}} \\
:= & A+B .
\end{aligned}
$$

Then

$$
A=\tilde{g}^{m l} \tilde{g}^{k n} g_{m p} \nabla^{p} \rho \frac{\partial^{2} \rho}{\partial u^{l} \partial u^{k}} g_{n q} \nabla^{q} \rho=\frac{1}{|x|^{4}} \nabla^{l} \rho \frac{\partial^{2} \rho}{\partial u^{l} \partial u^{k}} \nabla^{k} \rho
$$

and

$$
\begin{aligned}
B & =\frac{1}{2} \tilde{g}^{m l} \frac{\partial \rho}{\partial u^{m}} \frac{\partial \rho}{\partial u^{k}} \frac{\partial \rho}{\partial u^{n}} \frac{\partial}{\partial u^{l}}\left(\frac{\partial u^{k}}{\partial x^{j}}\right) \frac{\partial u^{n}}{\partial x^{j}}+\frac{1}{2} \tilde{g}^{m l} \frac{\partial \rho}{\partial u^{m}} \frac{\partial \rho}{\partial u^{n}} \frac{\partial \rho}{\partial u^{k}} \frac{\partial}{\partial u^{l}}\left(\frac{\partial u^{n}}{\partial x^{j}}\right) \frac{\partial u^{k}}{\partial x^{j}} \\
& =\frac{1}{2} \tilde{g}^{m l} \frac{\partial \rho}{\partial u^{m}} \frac{\partial \rho}{\partial u^{k}} \frac{\partial \rho}{\partial u^{n}} \frac{\partial}{\partial u^{l}}\left(\frac{\partial u^{k}}{\partial x^{j}} \frac{\partial u^{n}}{\partial x^{j}}\right) \\
& =\frac{1}{2} \tilde{g}^{m l} \frac{\partial \rho}{\partial u^{m}} \frac{\partial \rho}{\partial u^{k}} \frac{\partial \rho}{\partial u^{n}} \frac{\partial \tilde{g}^{k n}}{\partial u^{l}} .
\end{aligned}
$$

Now $B=B_{1}=B_{2}=B_{3}$, where

$$
\begin{aligned}
& B_{1}=\frac{1}{2} \tilde{g}^{m l} g_{m r} g_{k s} \frac{\partial \tilde{g}^{k n}}{\partial u^{l}} \frac{\partial \rho}{\partial u^{n}} \nabla^{r} \rho \nabla^{s} \rho=\frac{1}{2|x|^{2}} g_{k s} \frac{\partial \tilde{g}^{k n}}{\partial u^{r}} \frac{\partial \rho}{\partial u^{n}} \nabla^{r} \rho \nabla^{s} \rho, \\
& B_{2}=\frac{1}{2} \tilde{g}^{m l} g_{m s} g_{n r} \frac{\partial \tilde{g}^{\tilde{g}^{n}}}{\partial u^{l}} \frac{\partial \rho}{\partial u^{k}} \nabla^{r} \rho \nabla^{s} \rho=\frac{1}{2|x|^{2}} g_{n r} \frac{\partial \tilde{g}^{k n}}{\partial u^{s}} \frac{\partial \rho}{\partial u^{k}} \nabla^{r} \rho \nabla^{s} \rho, \\
& B_{3}=\frac{1}{2} \tilde{g}^{m l} g_{k r} g_{n s} \frac{\partial \tilde{g}^{k n}}{\partial u^{l}} \frac{\partial \rho}{\partial u^{m}} \nabla^{r} \rho \nabla^{s} \rho .
\end{aligned}
$$

Hence

$$
B=-\frac{1}{|x|^{4}} \Gamma_{r s}^{p} \frac{\partial \rho}{\partial u^{p}} \nabla^{r} \rho \nabla^{s} \rho,
$$


where $\Gamma_{r s}^{p}=-\frac{|x|^{2}}{2}\left(g_{k s} \frac{\partial \tilde{g}^{k p}}{\partial u^{r}}+g_{n r} \frac{\partial \tilde{g}^{p n}}{\partial u^{s}}-|x|^{2} \tilde{g}^{p l} g_{k r} g_{n s} \frac{\partial \tilde{g}^{k n}}{\partial u^{l}}\right)$. Using that $\frac{\partial}{\partial u^{r}}\left(g_{k s} \tilde{g}^{k p}\right)=$ $\frac{\partial}{\partial u^{r}}\left(\frac{1}{|x|^{2}} \delta_{s p}\right)=0$ (points $x \in \mathbb{R}^{n+1}$ are parameterized by $\beta(u)$ and $|x|$ ), we compute $g_{k s} \frac{\partial \tilde{g}^{k p}}{\partial u^{r}}=\frac{\partial}{\partial u^{r}}\left(g_{k s} \tilde{g}^{k p}\right)-\tilde{g}^{k p} \frac{\partial g_{k s}}{\partial u^{r}}=-\tilde{g}^{k p} \frac{\partial g_{k s}}{\partial u^{r}}$. This gives

$$
\begin{aligned}
\Gamma_{r s}^{p} & =\frac{|x|^{2}}{2}\left(\tilde{g}^{k p} \frac{\partial g_{k s}}{\partial u^{r}}+\tilde{g}^{k p} \frac{\partial g_{k r}}{\partial u^{s}}-|x|^{2} \tilde{g}^{p l} g_{k r} \tilde{g}^{k n} \frac{\partial g_{n s}}{\partial u^{l}}\right) \\
& =\frac{|x|^{2}}{2}\left(\tilde{g}^{k p} \frac{\partial g_{k s}}{\partial u^{r}}+\tilde{g}^{k p} \frac{\partial g_{k r}}{\partial u^{s}}-\tilde{g}^{p k} \frac{\partial g_{r s}}{\partial u^{k}}\right) .
\end{aligned}
$$

Since $|x|=1$, and therefore $\tilde{g}^{p k}=g^{k p}$, we have that on $\Gamma$

$$
\Gamma_{r s}^{p}=\frac{1}{2} g^{k p}\left(\frac{\partial g_{k s}}{\partial u^{r}}+\frac{\partial g_{k r}}{\partial u^{s}}-\frac{\partial g_{r s}}{\partial u^{k}}\right),
$$

which coincides with our definition for $\Gamma_{r s}^{p}$ at the beginning of Section 3

Equations (52), (53) and (54) and the relations Hess $\rho(V, W)=V^{i}(H e s s \rho)_{i j} W^{j}$ and $(H e s s \rho)_{i j}=\frac{\partial^{2} \rho}{\partial u^{i} \partial u^{j}}-\Gamma_{i j}^{k} \frac{\partial \rho}{\partial u^{k}}$ give (14). This finishes the proof of the lemma.

\section{Appendix B. Appendix B: Proof of Lemma 5}

In this appendix we prove Lemma [5. In what follows we drop the subindex $t$ in $S_{t}$, as well as the $t$-dependence of $\rho$. First, we note that if $\beta(u)$ is a local parametrization of $\Gamma$, then

$$
\tilde{\beta}(u)=\rho(\beta(u)) \beta(u)
$$

is a local parametrization of $S$. We denote metrics on $\Gamma$ and $S$ by $g_{i j}:=g_{i j}^{\Gamma}$ and $\tilde{g}_{i j}:=g_{i j}^{S}$, respectively. Let $g:=\operatorname{det}\left(g_{i j}\right)$ (not to be confused with the map $g(\rho)$ defined in (6) ) and $\tilde{g}:=\operatorname{det}\left(\tilde{g}_{i j}\right)$.

The following lemma proves a simple formula estabilishing the relation between $g$ and $\tilde{g}$.

LEMMA 10.

$$
\tilde{g}=\rho^{2 n-2} g\left(\rho^{2}+|\nabla \rho|^{2}\right) .
$$

PRoOF. The definitions $\tilde{g}_{i j}=\frac{\partial \tilde{\sigma}^{k}}{\partial u^{i}} \frac{\partial \tilde{\sigma}^{k}}{\partial u^{j}}$ and $g_{i j}=\frac{\partial \sigma^{k}}{\partial u^{i}} \frac{\partial \sigma^{k}}{\partial u^{j}}$, imply

$$
\tilde{g}_{i j}=\left(\frac{\partial \sigma^{k}}{\partial u^{i}} \rho+\sigma^{k} \frac{\partial \rho}{\partial u^{i}}\right)\left(\frac{\partial \sigma^{k}}{\partial u^{j}} \rho+\sigma^{k} \frac{\partial \rho}{\partial u^{j}}\right) .
$$

Since $\sigma \cdot \sigma=1$ on $\Gamma$ and therefore $\sigma \cdot \frac{\partial \sigma}{\partial u^{m}}=0$, (57) gives

and hence

$$
\tilde{g}_{i j}=g_{i j} \rho^{2}+\frac{\partial \rho}{\partial u^{i}} \frac{\partial \rho}{\partial u^{j}},
$$

$$
\tilde{g}=\operatorname{det}\left(g_{i j} \rho^{2}+\frac{\partial \rho}{\partial u^{i}} \frac{\partial \rho}{\partial u^{j}}\right) .
$$

Writing $\left(g_{i j} \rho^{2}+\frac{\partial \rho}{\partial u^{i}} \frac{\partial \rho}{\partial u^{j}}\right)=\rho^{2} G^{1 / 2}\left(\mathbf{1}+\rho^{-2} G^{-1 / 2}\left(\frac{\partial \rho}{\partial u^{i}}\right)\left(\frac{\partial \rho}{\partial u^{j}}\right) G^{-1 / 2}\right) G^{1 / 2}$, where $G:=\left(g_{i j}\right)$, we compute

$$
\tilde{g}=\rho^{2 n} g \operatorname{det}(\mathbf{1}+\lambda P),
$$

where $\lambda:=\rho^{-2}|\nabla \rho|^{2}$ and $P$ is the projection onto the vector $G^{-1 / 2}\left(\frac{\partial \rho}{\partial u^{j}}\right)$. Due to the relation $\operatorname{det}(1+\lambda P)=1+\lambda$, for any rank-one projection $P$, we arrive at (59). 
By the local definition of the integral over a surface, we have

$$
\int_{S} f=\int_{U} f(\rho(\beta(u)) \beta(u)) \sqrt{\tilde{g}} d^{n} u=\int_{\Gamma} f(\rho(\cdot) \cdot) \sqrt{\frac{\tilde{g}}{g}} .
$$

The last equation together with (59) and the definition (7) proves (15).

\section{Appendix C. Appendix C: Expression for $\mathcal{N}(\xi)$}

In this appendix we derive the explicit expression for the term $\mathcal{N}(\xi)$, defined in (27), with (17), (5) and (6). Let $\partial F(\rho)$ and $\left.\partial^{2} F(\rho)\right)$ denote the first and second Gâteaux derivatives of $F(\rho)$ evaluated at $\rho, \partial F(\rho): \xi \rightarrow \partial F(\rho) \xi$ and $\partial^{2} F(\rho)$ : $(\xi, \eta) \rightarrow \partial^{2} F(\rho)(\xi, \eta)$ and at $\xi$ and $\eta$. Recall the notation

$$
\mu(\rho)=\sqrt{\rho^{2}+|\nabla \rho|^{2}}
$$

and the definition (17) of the map $J$. In what follows we use the shorthand $f^{\prime \prime}(\xi, \eta) \equiv \operatorname{Hessf}(\xi, \eta)$. We have

Lemma 11. The nonlinearity $\mathcal{N}(\xi):=J\left(\rho_{\alpha}+\xi\right)-J\left(\rho_{\alpha}\right)-\partial J\left(\rho_{\alpha}\right) \xi$ can be written as

$$
\mathcal{N}(\xi)=\int_{0}^{1} d s \int_{0}^{s} d r \partial^{2} J\left(\rho_{\alpha}+r \xi\right)(\xi, \xi),
$$

where $\partial^{2} J(\rho)=\partial^{2} G(\rho)+\partial^{2} g(\rho)$, with $\partial^{2} G(\rho)$ and $\partial^{2} g(\rho)$ given by

$$
\begin{aligned}
& \partial^{2} G(\rho)(\xi, \xi)=-\frac{4 \xi \Delta \xi}{\rho^{3}}+6 \frac{\xi^{2} \Delta \rho}{\rho^{4}}-\frac{2 n \xi^{2}}{\rho^{3}} \\
& +\frac{2|\nabla \xi|^{2}}{\rho \mu^{2}}-\frac{2 \nabla \rho \nabla \xi(2 \rho \xi+5 \nabla \rho \nabla \xi)}{\rho \mu^{4}}+\frac{2|\nabla \rho|^{2} \xi(\rho \xi+2 \nabla \rho \nabla \xi)}{\rho^{2} \mu^{4}} \\
& -\frac{2 \nabla \rho \xi \nabla \xi}{\rho^{2} \mu^{2}}+\frac{2|\nabla \rho|^{2} \xi^{2}}{\rho^{3} \mu^{2}}+\frac{8|\nabla \rho|^{2} \nabla \rho \nabla \xi(\rho \xi+\nabla \rho \nabla \xi)}{\rho \mu^{6}} \\
& -\frac{2}{\rho^{2} \mu^{2}}\left(2 \xi^{\prime \prime}(\nabla \rho, \nabla \xi)+\rho^{\prime \prime}(\nabla \xi, \nabla \xi)\right) \\
& -2\left(\frac{3 \xi^{2}}{\rho^{4} \mu^{2}}+4 \frac{\xi(\rho \xi+\nabla \rho \nabla \xi)}{\rho^{3} \mu^{4}}+\frac{4(\rho \xi+\nabla \rho \nabla \xi) \xi}{\rho^{2} \mu^{6}}-\frac{\xi^{2}+|\nabla \xi|^{2}}{\rho^{2} \mu^{4}}\right) \rho^{\prime \prime}(\nabla \rho, \nabla \rho) \\
& +2\left(\frac{\xi}{\rho^{3} \mu^{2}}+\frac{\rho \xi+\nabla \rho \nabla \xi}{\rho^{2} \mu^{4}}\right)\left(2 \rho^{\prime \prime}(\nabla \rho, \nabla \xi)+\xi^{\prime \prime}(\nabla \rho, \nabla \rho)\right)
\end{aligned}
$$

and

$$
\begin{aligned}
\partial^{2} g(\rho)(\xi, \xi)= & {\left[\frac{-2 \mu}{\rho^{3}} \xi^{2}+2 \frac{\rho \xi^{2}+\nabla \rho \xi \nabla \xi}{\rho^{2} \mu}-\frac{\xi^{2}+\xi|\nabla \xi|}{\rho \mu}+\frac{(\rho \xi+\nabla \rho \nabla \xi)^{2}}{\rho \mu^{3}}\right] B } \\
& +2\left(\frac{\mu \xi}{\rho^{2}}-\frac{\rho \xi+\nabla \rho \nabla \xi}{\rho \mu}\right) \partial B \xi-\frac{\mu(\rho)}{\rho} \partial^{2} B(\xi, \xi),
\end{aligned}
$$

where

$$
\begin{array}{r}
B(\rho):=\frac{\int_{\Gamma} G(\rho) \rho^{n}}{\int_{\Gamma} \mu(\rho) \rho^{n-1}}, \partial B \xi=\frac{1}{\int_{\Gamma} \mu(\rho) \rho^{n-1}} \int_{\Gamma}\left(\partial G(\rho) \xi \rho^{n}+G(\rho) n \rho^{n-1} \xi\right) \\
-\frac{1}{\left(\int_{\Gamma} \mu(\rho) \rho^{n-1}\right)^{2}}\left(\int_{\Gamma} G(\rho) \rho^{n} \int_{\Gamma}\left(\partial \mu(\rho) \xi \rho^{n-1}+\mu(\rho)(n-1) \rho^{n-2} \xi\right)\right)
\end{array}
$$




$$
\begin{aligned}
& \partial^{2} B(\xi, \xi)=\frac{1}{\int_{\Gamma} \mu(\rho) \rho^{n-1}}\left(\int_{\Gamma} \partial^{2} G(\rho)(\xi, \xi) \rho^{n}\right. \\
& \left.+2 \int_{\Gamma} \partial G(\rho) \xi n \rho^{n-1} \xi+\int_{\Gamma} n(n-1) G(\rho) \rho^{n-2} \xi^{2}\right) \\
& -\frac{\int_{\Gamma} G(\rho) \rho^{n}}{\left(\int_{\Gamma} \mu(\rho) \rho^{n-1}\right)^{2}}\left(\int_{\Gamma} \partial^{2} \mu(\rho)(\xi, \xi) \rho^{n-1}\right. \\
& \left.+2 \int_{\Gamma} \partial \mu(\rho) \xi(n-1) \rho^{n-2} \xi+\int_{\Gamma}(n-1)(n-2) \mu(\rho) \rho^{n-3} \xi^{2}\right) \\
& -\frac{1}{\left(\int_{\Gamma} \mu(\rho) \rho^{n-1}\right)^{2}}\left(\int _ { \Gamma } \left(\partial G(\rho) \xi \rho^{n}\right.\right. \\
& \left.\left.+G(\rho) n \rho^{n-1} \xi\right) \int_{\Gamma}\left(\partial \mu(\rho) \xi \rho^{n-1}+\mu(\rho)(n-1) \rho^{n-2} \xi\right)\right) \\
& +2 \frac{1}{\left(\int_{\Gamma} \mu(\rho) \rho^{n-1}\right)^{3}}\left(\int _ { \Gamma } \left(\partial \mu(\rho) \xi \rho^{n-1}\right.\right. \\
& \left.\left.+\mu(\rho)(n-1) \rho^{n-2} \xi\right)\right)^{2} \int_{\Gamma} G(\rho) \rho^{n},
\end{aligned}
$$

with $\partial \mu(\rho) \xi=\frac{\rho \xi+\nabla \rho \nabla \xi}{\mu}, \partial^{2} \mu(\rho)(\xi, \xi)=\frac{\xi^{2}+\nabla \xi \nabla \xi}{\mu}-\frac{(\rho \xi+\nabla \rho \nabla \xi)^{2}}{\mu^{3}}$, (62) and

$$
\begin{aligned}
\partial G(\rho) \xi & =\frac{\Delta \xi}{\rho^{2}}-\frac{2 \Delta \rho}{\rho^{3}} \xi+\frac{n \xi}{\rho^{2}} \\
& +\frac{2 \nabla \rho \nabla \xi}{\rho \mu^{2}}-\frac{|\nabla \rho|^{2} \xi}{\rho^{2} \mu^{2}}-2 \frac{|\nabla \rho|^{2}(\rho \xi+\nabla \rho \nabla \xi)}{\rho \mu^{4}} \\
& -\frac{1}{\rho^{2} \mu^{2}}\left(2 \rho^{\prime \prime}(\nabla \rho, \nabla \xi)+\xi^{\prime \prime}(\nabla \rho, \nabla \rho)\right) \\
& +2\left(\frac{\xi}{\rho^{3} \mu^{2}}+\frac{\rho \xi+\nabla \rho \nabla \xi}{\rho^{2} \mu^{4}}\right) \rho^{\prime \prime}(\nabla \rho, \nabla \rho) .
\end{aligned}
$$

Proof. We write the nonlinearity $\mathcal{N}(\xi):=J\left(\rho_{\alpha}+\xi\right)-J\left(\rho_{\alpha}\right)-\partial J\left(\rho_{\alpha}\right) \xi$ in the standard form

$$
\mathcal{N}(\xi)=\int_{0}^{1} d s \int_{0}^{s} d r \partial_{r}^{2} J\left(\rho_{\alpha}+r \xi\right) .
$$

The definitions of the first and second Gâteaux derivatives imply

$$
\partial J(\rho) \xi=\left.\partial_{r} J(\rho+r \xi)\right|_{r=0},
$$

and

$$
\partial^{2} J(\rho)(\xi, \xi)=\left.\partial_{r}^{2} J(\rho+r \xi)\right|_{r=0} .
$$

Using the expressions above, we obtain the formula

$$
\mathcal{N}(\xi)=\int_{0}^{1} d s \int_{0}^{s} d r \partial^{2} J\left(\rho_{\alpha}+r \xi\right)(\xi, \xi) .
$$

We use $\partial^{2} J(\rho)=\partial^{2} G(\rho)+\partial^{2} g(\rho)$, and compute $\partial^{2} G(\rho)$ and $\partial^{2} g(\rho)$ separately. Using the definition

$$
G(\rho):=\frac{\Delta \rho}{\rho^{2}}-\frac{n}{\rho}+\frac{|\nabla \rho|^{2}}{\rho \mu^{2}}-\frac{\rho^{\prime \prime}(\nabla \rho, \nabla \rho)}{\rho^{2} \mu^{2}},
$$


we find (66) and (62).

Next, we use the definition of $g(\rho)$, which can be rephrased as $g(\rho)=-A(\rho) B(\rho)$, where $A(\rho):=\frac{\mu(\rho)}{\rho}$ and $B(\rho):=\frac{\int_{\Gamma} G(\rho) \rho^{n}}{\int_{\Gamma} \mu(\rho) \rho^{n-1}}$, as in the lemma. Using this representation and using $\partial^{2} g=-\partial^{2} A B-2 \partial A \partial B-A \partial^{2} B$ and

$$
\begin{gathered}
\partial \mu(\rho) \xi=\frac{\rho \xi+\nabla \rho \nabla \xi}{\sqrt{\rho^{2}+|\nabla \rho|^{2}}} \\
\partial^{2} \mu(\rho)(\xi, \xi)=\frac{\xi^{2}+|\nabla \xi|^{2}}{\sqrt{\rho^{2}+|\nabla \rho|^{2}}}-\frac{(\rho \xi+\nabla \rho \nabla \xi)^{2}}{\left(\rho^{2}+|\nabla \rho|^{2}\right)^{3 / 2}},
\end{gathered}
$$

we obtain (63).

Finally, using the definition of $B$ in (64), we find (64), and (65).

\section{Appendix D. Appendix D: Proof of (36)}

LEMMA 12. Let $k>\frac{n}{2}+1$ and assume that $|\xi| \leq \frac{1}{2} \rho_{\alpha}$. Then

$$
\left\|L_{R 0}^{\frac{k-1}{2}} \mathcal{N}(\xi)\right\| \lesssim\left(\Lambda_{k}^{1 / 2}(\xi)+\Lambda_{k}^{k}(\xi)\right)\left\|L_{R 0}^{\frac{k+1}{2}} \xi\right\| .
$$

Proof. Assume first that $k$ is an integer. Then $\left\|L_{R 0}^{\frac{k-1}{2}} \eta\right\| \simeq\|\eta\|_{H^{k-1}}^{2} \simeq\|\eta\|_{L^{2}}^{2}+$ $\left\|\nabla^{k-1} \eta\right\|_{L^{2}}^{2}$. Now, by Lemma 11]. $\left|\nabla^{k-1} \mathcal{N}(\xi)\right|$ is bounded above by terms of the form $\left|\xi^{t}(\nabla \xi)^{r}\left(\nabla^{\alpha_{1}} \xi\right) \cdots\left(\nabla^{\alpha_{s}} \xi\right)\right|$, where

$0 \leq t, r \leq k+1,1 \leq s \leq k, t+r+s \geq 2,2 \leq \alpha_{1} \leq \cdots \leq \alpha_{s} \leq k-s+2, \alpha_{1}+\cdots+\alpha_{s} \leq k+s$.

Note that the last two conditions in (73) imply that $s \leq k$. Then by Hölder's inequality we have

$$
\left\|\nabla^{k-1} \mathcal{N}(\xi)\right\| \leq\|\nabla \xi\|_{L^{\infty}}^{r}\left\|\nabla^{\alpha_{1}} \xi\right\|_{L^{p_{1}}} \cdots\left\|\nabla^{\alpha_{s}} \xi\right\|_{L^{p_{s}}},
$$

where $\frac{1}{p_{1}}+\cdots+\frac{1}{p_{s}}=\frac{1}{2}$.

Since $k>\frac{n}{2}+1$, we have, by the Sobolev embedding theorem, that $\|\xi\|_{L^{\infty}}+$ $\|\nabla \xi\|_{L^{\infty}} \lesssim\|\xi\|_{H^{k}}$. Moreover, we choose $p_{i}$ so that $k-\alpha_{i}>\frac{n}{2}-\frac{n}{p_{i}}$ for all $i=$ $1, \cdots, s-1$ and $k+1-\alpha_{s}>\frac{n}{2}-\frac{n}{p_{s}}$ (this choice implies $\sum_{j=1}^{s} \alpha_{j}<\frac{n}{2}+1+\left(k-\frac{n}{2}\right) s$, which is compatible with (73) ). Then, using the Sobolev embedding theorem again, we have $\left\|\nabla^{\alpha_{i}} \xi\right\|_{L^{p_{i}}} \leq\|\xi\|_{H^{k}}$, for $i=1, \cdots, s-1$, and $\left\|\nabla^{\alpha_{s}} \xi\right\|_{L^{p_{s}}} \leq\|\xi\|_{H^{k+1}}$. Combining these estimates gives us

$$
\left\|L_{R 0}^{\frac{k-1}{2}} \mathcal{N}(\xi)\right\| \lesssim\|\xi\|_{H^{k}}^{r+s-1}\|\xi\|_{H^{k}} .
$$

Now from $1 \leq r+s-1 \leq 2 k$ and Proposition 8 we obtain (72). Furthermore, one can easily check that $k$ can be taken arbitrary close to $\frac{n}{2}+1$ (this means that one is able to satisfy $1 \geq \alpha_{i}-\frac{n}{p_{i}}$, for $i=1, \cdots, s-1,2 \geq \alpha_{s}-\frac{n}{p_{s}}$ and $\left.\alpha_{i} \geq 2, \forall i\right)$.

If $k$ is not integer, we proceed as follows. Let $\beta=k-[k] \in(0,1)$. We use the space $\tilde{H}^{\beta}$ with the norm

$$
\|f\|_{\tilde{H}^{\beta}}=\|f\|_{L^{2}}+\int \frac{d h}{|h|^{n+\beta}}\left\|\Delta_{h} f\right\|_{L^{2}},
$$

where $\Delta_{h} f(x)=f(x+h)-f(x)$. We have the embeddings

$$
\|f\|_{H^{\beta}} \lesssim\|f\|_{\tilde{H}^{\beta}} \lesssim\|f\|_{H^{\beta^{\prime}}}, \beta<\beta^{\prime} .
$$


Let us prove the first embedding:

$$
(-\Delta+1)^{\beta / 2} f(x)=C_{\beta} f(x)+\int(f(x-y)-f(x)) G_{\beta}(y) d y,
$$

where $C_{\beta}$ is an analytic continuation of $C_{\beta}:=\int G_{\beta}(x) d x$ with $\operatorname{Re}(\beta)<n$ and $G_{\beta}(y):=\int e^{i y \cdot k}\left(|k|^{2}+1\right)^{\beta / 2} d k$. Note that $G_{\beta}(y) \sim|y|^{-n-\beta}$ as $|y| \rightarrow 0$ and is exponentially decaying at $\infty$. So

$$
\|f\|_{H^{\beta}}=\left\|(-\Delta+1)^{\beta / 2} f\right\|_{L^{2}} \leq C_{\beta}\|f\|_{L^{2}}+\int \frac{d y}{|y|^{n+\beta}}\left\|\Delta_{y} f\right\|_{L^{2}} \lesssim\|f\|_{\tilde{H}^{\beta}},
$$

which proves the first embedding in (74).

For the second embedding, let $\varphi=(-\Delta+1)^{\beta^{\prime} / 2} f$. Then

$$
f=(-\Delta+1)^{-\beta^{\prime} / 2} \varphi=\int \tilde{G}_{\beta^{\prime}}(x-y) \varphi(y) d y,
$$

where $\tilde{G}_{\beta^{\prime}}(y):=\int e^{i y \cdot k}\left(|k|^{2}+1\right)^{-\beta^{\prime} / 2} d k$. Note that $\tilde{G}_{\beta^{\prime}}(y) \sim|y|^{-n+\beta^{\prime}}$ as $|y| \rightarrow 0$ and is exponentially decaying at $\infty$. Let $\beta<\beta^{\prime \prime}<\beta^{\prime}$. Then

$$
\begin{aligned}
& \int_{|h| \leq 1} \frac{d h}{h \mid n+\beta}\left\|\Delta_{h} f\right\|_{L^{2}} \\
= & \int_{|h| \leq 1} \frac{d h}{|h|^{n+\beta}} \| \int_{|x-y| \leq 2}\left(\tilde{G}_{\beta^{\prime}}(x+h-y)-\tilde{G}_{\beta^{\prime}}(x-y)\right) \varphi(y) d y \\
& +\int_{|x-y| \geq 2}\left(\tilde{G}_{\beta^{\prime}}(x+h-y)-\tilde{G}_{\beta^{\prime}}(x-y)\right) \varphi(y) d y \|_{L^{2}} \\
\lesssim & \int_{|h| \leq 1} \frac{\overrightarrow{d h}}{|h| n+\beta}\left(|h|^{\beta^{\prime \prime}}\left\|\int_{|x-y| \leq 2}|x-y|^{-n+\beta^{\prime}-\beta^{\prime \prime}}|\varphi(y)| d y\right\|_{L^{2}}\right. \\
& \left.+|h|\left\|\int_{|x-y| \geq 2}|x-y|^{-n+\beta^{\prime}-1}|\varphi(y)| d y\right\|_{L^{2}}\right) \\
\lesssim & \|\varphi\|_{L^{2}}=\|f\|_{H^{\beta^{\prime}}}
\end{aligned}
$$

and

$$
\int_{|h| \geq 1} \frac{d h}{|h|^{n+\beta}}\left\|\Delta_{h} f\right\|_{L^{2}} \leq 2\|f\|_{L^{2}} \int_{|h| \geq 1} \frac{d h}{|h|^{n+\beta}} \lesssim\|f\|_{H^{\beta^{\prime}}} .
$$

This proves the second embedding in (74).

Using (74), we obtain

$$
\begin{aligned}
\left\|\prod_{j=1}^{s} \xi_{j}\right\|_{H^{\beta}} & \lesssim \int \frac{d h}{|h|^{n+\beta}}\left\|\Delta_{h} \prod_{j=1}^{s} \xi_{j}\right\|_{2} \\
& \leq \sum_{i=1}^{s} \int \frac{d h}{|h|^{n+\beta}}\left\|\prod_{j=1}^{i-1} \xi_{j} \Delta_{h} \xi_{i} \prod_{j=i+1}^{s} T_{h} \xi_{j}\right\|_{2} \\
& \leq \sum_{i=1}^{s}\left(\prod_{j \neq i}\left\|\xi_{j}\right\|_{p_{j}^{(i)}}\right) \int \frac{d h}{|h|^{n+\beta}}\left\|\Delta_{h} \xi_{i}\right\|_{p_{i}^{(i)}},
\end{aligned}
$$

where $T_{h} f(x)=f(x+h), \sum_{j=1}^{s} \frac{1}{p_{j}^{(i)}}=\frac{1}{2}$. Using appropriate embeddings, we conclude finally that

$$
\left\|\prod_{j=1}^{s} \xi_{j}\right\|_{H^{\beta}} \lesssim \sum_{i=1}^{s} \prod_{j=1}^{s}\left\|\xi_{j}\right\|_{H^{c_{j}^{(i)}}}
$$

where $c_{j}^{(i)}>\frac{n}{2}-\frac{n}{p_{j}^{(i)}} \forall j \neq i$ and $c_{i}^{(i)}-\beta>\frac{n}{2}-\frac{n}{p_{i}^{(i)}}$. Similarly as before we know that $\sum_{j=1}^{s} c_{j}^{(i)}-\beta>\frac{n}{2}(s-1)$, which guarantees the existence of $p_{j}^{(i)}$.

For $k$ not an integer, we write

$$
\|\mathcal{N}(\xi)\|_{H^{k-1}} \sim\left\|(-\Delta+1)^{\beta / 2} \nabla^{m} \mathcal{N}(\xi)\right\|_{L^{2}},
$$

where $m=[k]-1$ and $\beta=k-[k] \in(0,1) . \nabla^{m} \mathcal{N}(\xi)$ is treated as before to obtain

$$
\nabla^{m} \mathcal{N}(\xi) \sim \xi^{t}(\nabla \xi)^{r} \nabla^{\alpha_{1}} \xi \cdots \nabla^{\alpha_{s}} \xi
$$


where $t \leq m+2, r \leq m+2,2 \leq \alpha_{j} \leq m-s+3, \sum_{j=1}^{s} \alpha_{j} \leq m+1+s, s \leq m+1$ and $t+r+s \geq 2$.

If $\alpha_{j}<m+2 \forall j$, then, using (76) with $\xi_{j}=\nabla^{\alpha_{j}} \xi \forall j, c_{j}^{(i)}+\alpha_{j}=k \forall j \neq i$ and $c_{i}^{(i)}+\alpha_{i}=k+1$, we find

$$
\left\|\xi^{t}(\nabla \xi)^{r} \prod_{j=1}^{s} \nabla^{\alpha_{j}} \xi\right\|_{H^{\beta}} \lesssim\|\xi\|_{H^{k}}^{r+s-1}\|\xi\|_{H^{k+1}}
$$

We use this estimate, together with (77) and (78), to obtain

$$
\|\mathcal{N}(\xi)\|_{H^{k-1}} \lesssim \sum_{i=1}^{2[k]}\|\xi\|_{H^{k}}^{i}\|\xi\|_{H^{k+1}} .
$$

If $\alpha_{s}=m+2$ and therefore $s=1$, then we let $f=\xi^{t}(\nabla \xi)^{r}$ and proceed as

$$
(-\Delta+1)^{\beta / 2} f \nabla^{m+2} \xi=f(-\Delta+1)^{\beta / 2} \nabla^{m+2} \xi+\left[(-\Delta+1)^{\beta / 2}, f\right] \nabla^{m+2} \xi .
$$

The first term on the r.h.s. is easy to estimate:

$$
\begin{aligned}
& \left\|f(-\Delta+1)^{\beta / 2} \nabla^{m+2} \xi\right\| \leq\|f\|_{\infty}\|\xi\|_{H^{k+1}} \\
\leq & \|\xi\|_{H^{k}}^{t+r}\|\xi\|_{H^{k+1}} \leq \sum_{p=1}^{2}\|\xi\|_{H^{k}}^{p}\|\xi\|_{H^{k+1}} .
\end{aligned}
$$

To estimate the second term in the r.h.s. we note that

$$
\begin{aligned}
& {\left[(-\Delta+1)^{\beta / 2}, f\right] \eta=\int(f(x)-f(y)) G_{\beta}(x-y) \eta(y) d y } \\
= & \int \eta(x-z)(f(x-z)-f(x)) G_{\beta}(z) d z .
\end{aligned}
$$

Using this representation we obtain for $\beta^{\prime}>\beta$,

$$
\begin{aligned}
& \left\|\left[(-\Delta+1)^{\beta / 2}, f\right] \eta\right\|_{2} \\
\leq & \sup _{z}\left\|\eta(\cdot-z) \frac{f(\cdot-z)-f(\cdot)}{|z|^{\beta^{\prime}}}\right\|_{L^{2}(d x)} \int|z|^{\beta^{\prime}}\left|G_{\beta}(z)\right| d z \\
\lesssim & \sup _{z}\left\|\eta(\cdot-z) \frac{f(\cdot-)-f(\cdot)}{\left.|z|\right|^{\beta^{\prime}}}\right\|_{L^{2}(d x)} \\
\leq & \|\eta\|_{q} \sup _{z}\left\|\frac{\Delta_{z} f}{\left.|z|\right|^{\beta^{\prime}}}\right\|_{p},
\end{aligned}
$$

where $\frac{1}{p}+\frac{1}{q}=\frac{1}{2}$. Similar to (75), we have

$$
\sup _{z}\left\|\frac{1}{|z|^{\gamma}} \Delta_{z} f\right\|_{H^{b}} \lesssim\|f\|_{H^{b+\gamma^{\prime}}}, \gamma^{\prime}>\gamma .
$$

Using this estimate and Sobolev embedding theorem, we find

$$
\left\|\left[(-\Delta+1)^{\beta / 2}, f\right] \eta\right\|_{2} \lesssim\|\eta\|_{H^{a}} \sup _{z}\left\|\frac{\Delta_{z} f}{|z|^{\beta^{\prime}}}\right\|_{H^{b}} \lesssim\|\eta\|_{H^{a}}\|f\|_{H^{b+\beta^{\prime \prime}}},
$$

where $\beta^{\prime \prime}>\beta^{\prime}, a>\frac{n}{2}-\frac{n}{q}, b>\frac{n}{2}-\frac{n}{p}$. Taking $f=\xi^{t}(\nabla \xi)^{r}$ and $\eta=\nabla^{m+2} \xi, a=\beta$, we find

$$
\left\|\left[(-\Delta+1)^{\beta / 2}, f\right] \nabla^{m+2} \xi\right\| \leq\left\|\xi^{t}(\nabla \xi)^{r}\right\|_{H^{r+\beta^{\prime \prime}}}\|\xi\|_{H^{k+1}} .
$$

Note that $\beta^{\prime \prime}+r>n-\frac{n}{2}=\frac{n}{2}$. Let $\beta^{\prime \prime}+r=j$. As before, we estimate

$$
\left\|\xi^{t}(\nabla \xi)^{r}\right\|_{H^{j}} \lesssim \sum_{j_{1}+\cdots+j_{t+r}=j}\left\|\nabla^{j_{1}} \xi \cdots \nabla^{j_{t}} \xi \nabla^{j_{t+1}+1} \xi \cdots \nabla^{j_{t+r}+1} \xi\right\|_{2} \lesssim\|\xi\|_{H^{j+1}}^{t+r} \forall j>\frac{n}{2} .
$$

Since $k>\frac{n}{2}+1$, we can take $j=k-1$ and so

$$
\left\|\left[(-\Delta+1)^{\beta / 2}, f\right] \nabla^{m+2} \xi\right\| \leq\|\xi\|_{H^{k}}^{t+r}\|\xi\|_{H^{k+1}},
$$

where, recall, $f=\xi^{t}(\nabla \xi)^{r}$. This inequality together with (77), (81) and (82) implies (80) also in this case. As was mentioned above (80) implies (72). 


\section{References}

[1] N. D. Alikakos And A. Freire, The normalized mean curvature flow for a small bubble in a Riemannian manifold. J. Differential Geom., 64(2), pp. 247-303, 2003.

[2] B. Andrews, Volume-preserving anisotropic mean curvature flow, Indiana Univ. Math. J., 50 (2): 783-827, 2001.

[3] M. Athanassenas, Volume-preserving mean curvature flow of rotationally symmetric surfaces. Comment. Math. Helv., 72(1), pp. 52-66, 1997.

[4] M. Athanassenas, Behaviour of singularities of the rotationally symmetric, volumepreserving mean curvature flow. Calc. Var. Partial Differential Equations, 17(1), pp. 1-16, 2003.

[5] Lia Bronsard and Barbara Stoth, Volume-Preserving Mean Curvature Flow as a Limit of a Nonlocal Ginzburg-Landau Equation. SIAM J. Math. Anal., Volume 28, Issue 4, pp. 769-807, 1997.

[6] I. Chavel, Eigenvalues in Riemannian Geometry, volume 115 of Pure and Applied Mathematics. Academic Press Inc., Orlando, FL, 1984.

[7] Xinfu Chen, D. Hilhorst, E. Logak, Mass conserved Allen-Cahn equation and volume preserving mean curvature flow, arXiv:0902.3625v1, 2009.

[8] J. Escher and G. Simonett, A center manifold analysis for the Mullins-Sekerka model, J. Differential Eq., 143, pp. 267-292, 1998.

[9] J. Escher and G. Simonett, The volume preserving mean curvature flow near spheres, Proc. Amer. Math. Soc., 126(9), pp. 2789-2796, 1998.

[10] M. GAGe, On an area-preserving evolution equation for plane curves, Nonlinear Problems in Geometry, D. M. DeTurck, editor, Contemp. Math. 51, AMS, Providence, pp. 51-62, 1986.

[11] Zh. HuANG AND B. WANG, Geometric evolution equations and foliation on quasi-Fuchsian three-manifolds, arXiv:0907.2899v2, 2009.

[12] G. Huisken, The volume preserving mean curvature flow, J. Reine Angew. Math., 382, pp. 35-48, 1987.

[13] G. Huisken And S.T. YAU, Definition of center of mass for isolated physical systems and unique folliation by stable spheres with constant mean curvature flow, Invent. Math., 124 (1996), pp. 281-311.

[14] W.Kong And I.M. Sigal, On collapse of surfaces under mean curvature flow, in preparation.

[15] E. Kuwert And R. Schätzle, The Willmore of with small initial energy, J. Differential Geom. 57 (2001)), 409-441.

[16] W. KüHNEL, Differential Geometry, AMS, 2005.

[17] J. Rubinstein And P.Sternberg, Nonlocal reaction diffusion equations and nucleation, IMA J. Appl. Math., 48 (1992), pp. 249-264.

[18] E. M. Stein And G. Weiss, Introduction to Fourier Analysis on Euclidean Spaces. Princeton University Press, Princeton, N.J., 1971. Princeton Mathematical Series, No. 32.

Department of Applied Mathematics, University of Crete, and iACM/FORTH, Heraklion, Crete, Greece

Department of Applied Mathematics, University of Crete, and iACM/FORTH, Heraklion, Crete, Greece

Department of Mathematics, University of Toronto, Toronto, Canada

E-mail address: im.sigal@utoronto.ca 